\title{
Optimal Camera Placement for Motion Capture Systems in the Presence of Dynamic Occlusion
}

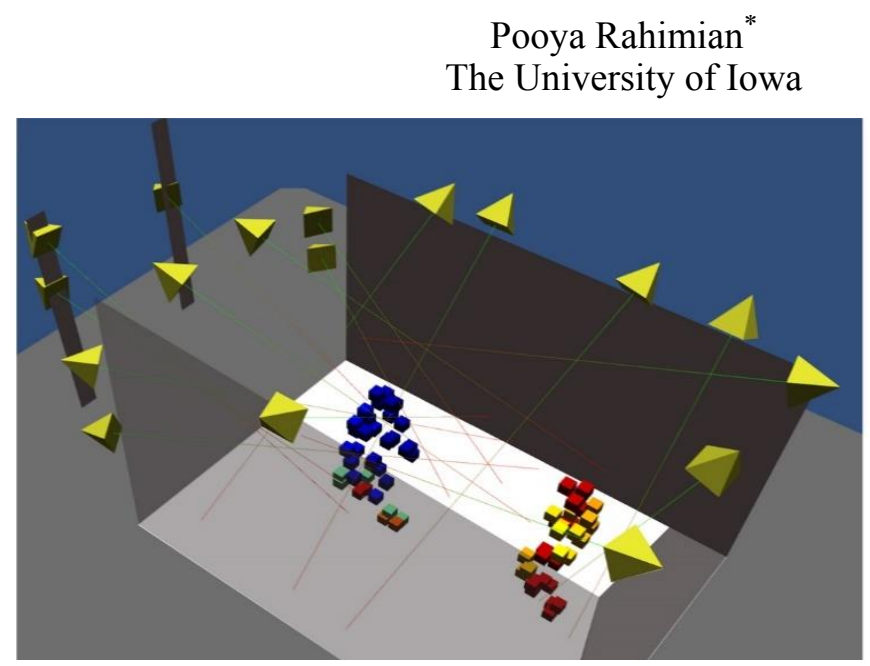

\author{
Joseph K. Kearney† \\ The University of Iowa
}

Figure 1: Visibility of target points from motion capture of a walker for two different camera configurations. Red points indicate low visibility; blue points indicate high visibility.

\begin{abstract}
Optical motion capture is based on estimating the threedimensional positions of markers by triangulation from multiple cameras. Successful performance depends on points being visible from at least two cameras and on the accuracy of the triangulation. Triangulation accuracy is strongly related to the positions and orientations of the cameras. Thus, the configuration of the camera network has a critical impact on performance. A poor camera configuration may result in a low quality three-dimensional (3D) estimation and consequently low quality of tracking. This paper proposes a camera configuration metric that is based on a probabilistic model of target point visibility from cameras with "good" views. An efficient algorithm, based on simulated annealing, is introduced for estimating the optimal configuration of an arbitrary set of cameras for a given distribution of target points. The accuracy and robustness of the algorithm are evaluated through both simulation and empirical measurement. An implementation of the method is available for download as a tool for the community [Rahimian and Kearney 2015] (Figure 1).
\end{abstract}

CR Categories: I.3.7 [Three-Dimensional Graphics and Realism]: Virtual reality. I.3.8 [Applications], B.6.3 [Design Aids]: Optimization and Simulation. G.1.6 [Optimization]: Simulated annealing

Keywords: Camera placement, CAVE, Motion capture, Dynamic occlusion, Virtual reality, Augmented reality.

\footnotetext{
* e-mail: Pooya-Rahimian@uiowa.edu

† e-mail: Joe-Kearney@uiowa.edu

Permission to make digital or hard copies of all or part of this work for personal or classroom use is granted without fee provided that copies are not made or distributed for profit or commercial advantage and that copies bear this notice and the full citation on the first page. Copyrights for components of this work owned by others than ACM must be first page. Copyrights for components of this work owned by others than ACM must be
honored. Abstracting with credit is permitted. To copy otherwise, or republish, to post on servers or to redistribute to lists, requires prior specific permission and/or a fee.

Request permissions from Permissions@acm.org.

VRST '15, November $13-15,2015$, Beijing, China.

(C) 2015 ACM. ISBN 978-1-4503-3990-2/15/11 . \$15.00

DOI: http://dx.doi.org/10.1145/2821592.2821596
}

\section{Introduction}

Robust, accurate motion tracking is a key component in most augmented and virtual reality systems. Motion tracking is used to estimate the location and orientation of the viewer's head in order to determine the eyepoint for correct perspective rendering of images. In addition, full-body motion tracking is frequently used to generate avatars that represent the viewer in the virtual environment. Optical motion tracking is a common technique for both head tracking and full-body motion capture. Cameras are placed around the area within which users are free to move. Feature points (typically reflective passive markers or active, light-emitting markers) are extracted from camera images and then the threedimensional locations of the markers are determined by triangulation from their locations in multiple images. The quality of the results depends greatly on the number and configuration of the cameras. This paper focuses on an automatic method to place a set of cameras in CAVE [Neira et al. 1992] and arena virtual reality systems taking into account the two primary sources of error related to camera layout: Marker visibility and triangulation accuracy.

The topology of the CAVE constrains the placement of tracking cameras. For instance, the screens occlude the views of cameras located outside the CAVE. Moreover, cameras cannot be placed inside the CAVE volume in front of the screens without disrupting immersion. This makes tracking of markers near the screens difficult, especially when the user's body occludes markers from cameras placed away from the screen.

This paper proposes a method to position a set of cameras for a given workspace to maximize the visibility of points with minimal $3 \mathrm{D}$ reconstruction error in the presence of dynamic occlusion. In many augmented and virtual realities systems, the likely target locations are known. For example, in our application, we ask participants to walk across a single-lane road in a virtual neighborhood. The path they take is aligned with the mid-line of the CAVE volume. Thus, with high probability, the targets will be located near the center of the volume. The method we propose 
estimates the best set of views for a specified set of target points. For this, we consider all possible locations of occluders and determine whether or not each target point is visible in at least two triangulable views (i.e. two views with sufficiently different viewing angles to allow for accurate triangulation).

The method allows a user to specify the number of cameras and possible camera locations by a set of constraints on camera positions and orientations. Cameras can be constrained to lie on a line segment, on a planar area, or in a volumetric region. Line, plane, and volume constraints can be combined to allow a rich set of possibilities for camera placement.

We demonstrate the method by determining the optimal locations for 17 cameras tracking motion in 4-screen CAVE. Permissible camera locations include a volume near the screen borders and two planar mounting surfaces at the open end of the CAVE. We compute optimal camera placements for three sets of target points: a uniform grid, a random sample of points from a normal distribution centered on the midline of the CAVE, and a set of points from full-body motion capture of a virtual road crossing in our pedestrian simulator. Finally, we empirically test the method by comparing the quality of tracking for an ad hoc placement of cameras and a placement based on optimising for visibility without considering triangulation error to the results of tracking with cameras placed to maximize visibility and minimize triangulation error.

\section{Related work}

Prior research on camera placement has focused on two factors that contribute to inaccurate reconstruction: (1) Placing cameras to minimize the error due to inaccurate triangulation and (2) Placing cameras to provide the best views in the presence of occlusion. Sanders-Reed [Sanders-Reed 2001] analyzed error propagation for $3 \mathrm{D}$ position estimation through triangulation of two cameras. The analysis points to the critical role that camera configuration has on error propagation - the optimal configuration is to have a 90 degree angle between the sensors. Error propagation increases to a maximum at 0 and 180 degrees. Olague $\&$ Mohr [Olague and Mohr 2002] analyzed the characteristics of error propagation in triangulation and verified the results experimentally. They introduced a criterion of error propagation by finding the maximum element in the diagonal of covariance $3 \mathrm{D}$ point reconstruction matrix. The maximum element represented the worst error propagation for a given configuration. In order to globally optimize the solution, a genetic algorithm was utilized. Olague et al. assumed cameras were mounted on a plane at a constant distance from the center point of target and also cameras were oriented to the target point. These two strong assumptions were relaxed in [Katz et al. 2010].

Cowan and Kovesi identified a set of constraints affecting camera network quality to avoid trial-and-error fashion camera placement [Cowan and Kovesi 1988]. They decomposed the viewed object into its surfaces. The solution for each surface was calculated separately based on a set of constraints and then local solutions were combined into a global solution for the original object. For each constraint (including resolution degradation, focus, and occlusion), they find a $3 \mathrm{D}$ volume where any point in that region satisfies the corresponding constraint. They then found the intersection of those individual 3D volumes to find a region(s) which can satisfy all the constraints. Mason [Mason 1997] expanded this idea with more constraints and proposed a heuristic model to find the optimal configuration.
The model proposed by Cowan and Kovesi only addresses stationary occlusion (self-occlusion). Mason's heuristic method finds a solution for a particular target but it may not be the optimal solution. In addition, Mason's method does not work for moving targets. Instead of dealing with object surfaces, Wu, Sharma, and Huang [Wu et al. 1998] consider the intersection of the projection pyramids to present a numerical measurement of uncertainty bounds.

Chen \& Davis [Chen and Davis 2008] developed a probabilistic model of occlusion. The occluder was assumed as an infinite plane rotating around the target point with a uniform distribution. The model predicts how likely a target point is to be visible by at least two cameras. Chen et al. introduced a quality metric of camera configuration in the presence of occlusion. Although [Chen and Davis 2008] nicely accounts for dynamic occlusion in the quality metric, they did not take into account the convergence angles of the cameras. As a consequence, the method can find configurations that produce large errors in target locations as a result of poor triangulation caused by having nearly parallel cameras.

This study leverages the strength of analytical studies [Nurre and Hall 1986; Olague and Mohr 2002; Sanders-Reed 2001; SandersReed 2002] in order to deal with error propagation in triangulation and exploits the benefits of a probabilistic model of occlusion [Chen and Davis] to introduce a comprehensive metric for camera configuration.

\section{Methodology}

The proposed method addresses three sources of motion capture failure: 3D reconstruction error, resolution degradation due to the distance from camera, and dynamic occlusion. The quality metric is then incorporated into a simulated annealing algorithm to minimize the residual of a given set of target points and workspace topology. The following sections present the $3 \mathrm{D}$ reconstruction error, resolution degradation, a probabilistic occlusion model, and a quality metric.

\subsection{D Point Reconstruction}

Stereo image reconstruction is based on triangulation from two or more cameras in known locations [Brown et al. 2003; Hartley and Sturm 1997; Liu et al. 2005; Wu, Sharma and Huang 1998]. The

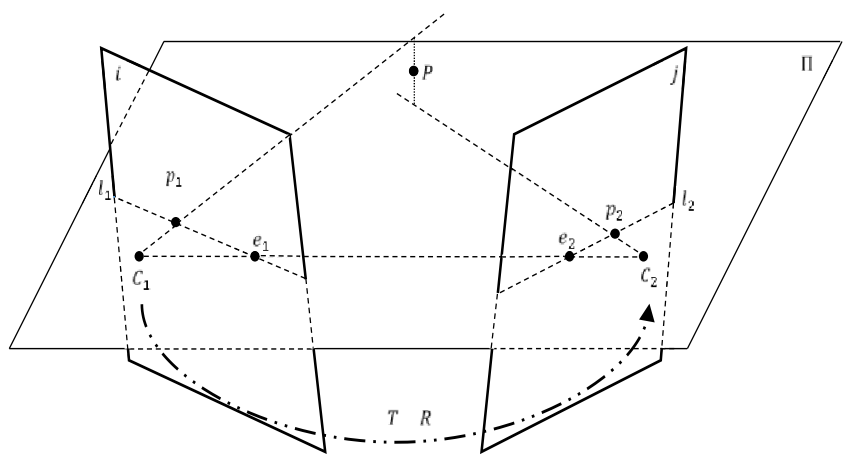

Figure 2: The $3 D$ location of a point $P$ can be computed by triangulation from the projections of $P$ onto two images. 


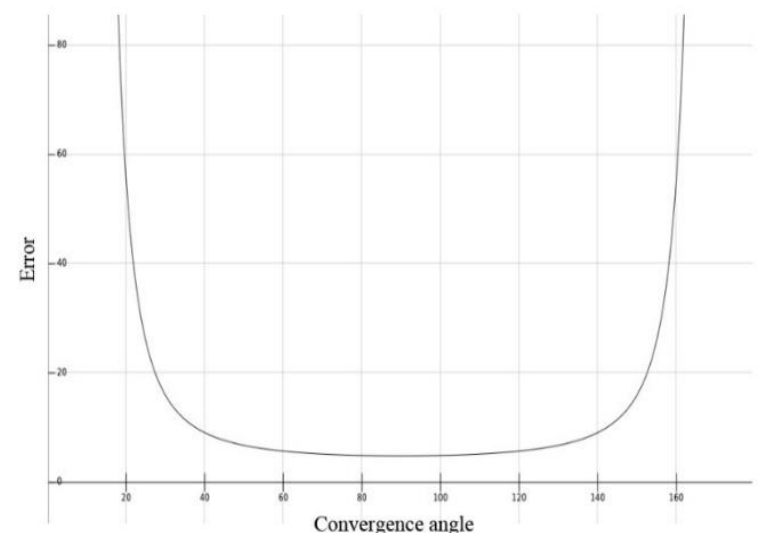

Figure 3: Error propagation in triangulation as a function of the convergence angle between two cameras based on [Olague \& Mohr 2002].

3D location of a target lies on a ray from the camera center of projection through the location of the target point on the image. When the target is identified in two non-parallel image planes, the target lies at the intersection of rays from the two camera centers of projection. Because of errors in estimates of camera rotation and translation parameters (i.e. the camera model), and inaccuracy in the estimates of the image coordinates, the two back-projected rays will not, in general, intersect exactly at the actual target position. Therefore, the target location is typically approximated, for example, by finding the midpoint between rays [Hartley and Zisserman 2003; Hartley and Sturm 1997] (Figure 2).

The accuracy of the computed 3D location of $P$ will depend on errors in the camera models (intrinsic parameters), errors in the estimates of the target's locations in the two images (extrinsic parameters), and on the error propagation characteristics of the linear system [Hartley and Zisserman 2003]. If the two camera view vectors (the rays from the centers of projection to the target point) are parallel, the linear system is singular and no solution can be found. If the view vectors are nearly parallel, the linear system will be poorly conditioned. This will cause inevitable small errors in the camera models or estimates of the locations of the target in the images to be magnified in the result.

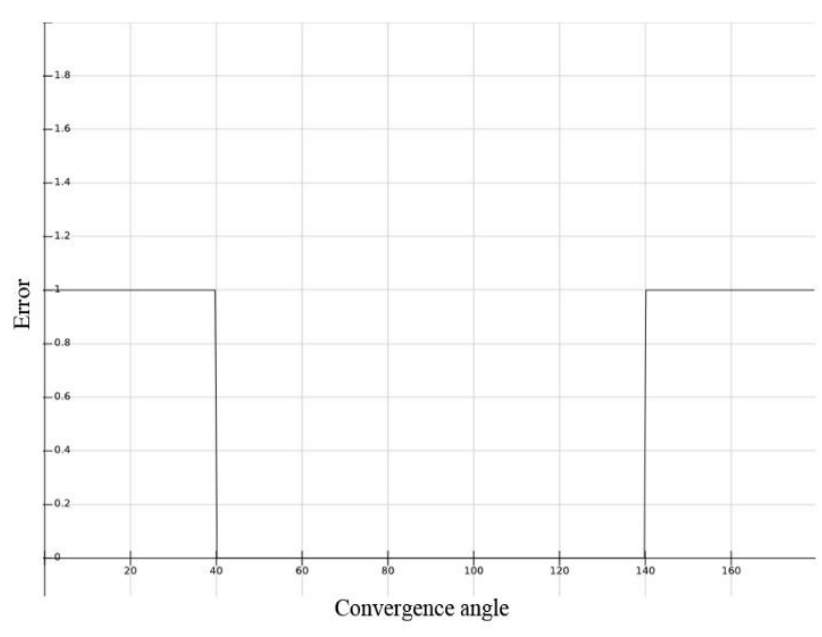

Figure 4: A simplified metric for error propagation in triangulation.

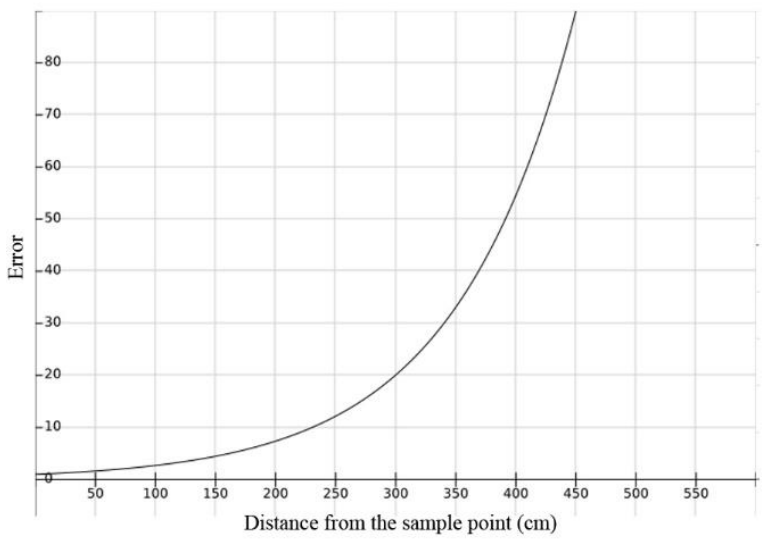

Figure 5: Error due to increasing distance between camera and target point based on [Wu, Sharma and Huang 1998].

Error propagation in 3D triangulation has been well studied [Olague and Mohr, 2002; Lourakis and Argyros 2004; SandersReed 2001; Sanders-Reed 2002; Sanders-Reed 2002]. Olague and Mohr measured error propagation in 3D triangulation from two cameras for a range of convergence angles. They calculated the uncertainty of a triangulated point using the Levenberg-Marquardt method. Figure 3 shows how error increases exponentially for parallel or nearly parallel convergence angles whereas the error is negligible for a range of angles about the perpendicular view.

To assure that the triangulation is well conditioned, we require that a target be in view of at least two cameras with view vectors that are sufficiently non-parallel that triangulation computations are well-conditioned. Based on the results of Olague and Mohr, we established a relatively conservative range for the acceptable convergence angle between two cameras at 40 and 140 degrees (Figure 4). When a target is visible in two cameras with view vectors in this range, we say the cameras have triangulable views.

\subsection{Resolution Degradation}

The other factor we consider in determining the visibility of a target is resolution degradation resulting from the distance between target point and camera. In the theory of triangulation, the target

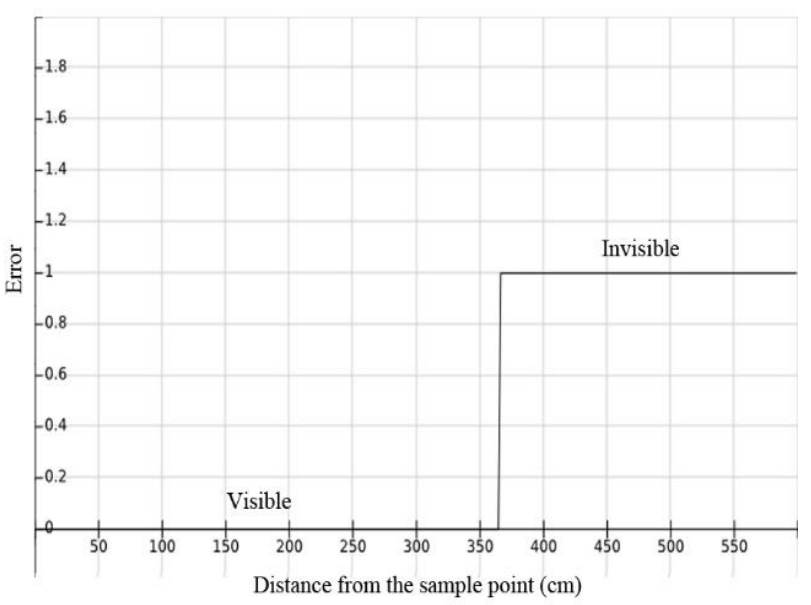

Figure 6: The camera range is split into a visible range where the camera effectively detects the target and invisible range where camera cannot reliably resolve the target. 
point is always represented by a single point. However in the real world, targets have volume. Spherical markers are typically used and the center point of projected image is used as the location of the target. The size of projection of the target onto the image plane shrinks as the target becomes more distant from the camera until it vanishes. Therefore, for any size of reflective marker and any type of camera, there is a distance where marker cannot be visible by the camera beyond that point. In [Wu, Sharma and Huang 1998], the error volume is estimated for a target as a function of the target's distance from the camera. Our model simplifies this error model by classifying visible or not-visible based on the distance from camera.

Figure 5 accounts for resolution degradation due to the target moving away from a pair of parallel cameras. In our study, our experiments revealed the effective range of camera is $\sim 14$ feet. Therefore, 12 feet $(\sim 365 \mathrm{~cm})$ was used as a safe range for the camera and any point beyond that was treated as invisible (Figure 6). This visibility threshold can be varied from one camera to other.

\subsection{Probabilistic Occlusion Modeling}

Occlusion modelling can be formulated as a visibility problem if the occluders are static and in known locations. The visibility problem (e.g. Art Gallery problem) is well-studied and there are a number of published methods to choose optimal vantage points [Fleishman et al. 1999; O'rourke 1987; Shermer 1992]. However, for most VR and AR applications, the occluders are dynamic and unpredictable. Occlusion may be caused by other users in the tracked space or by the participant's own body.

Chen \& Davis [Chen and Davis 2008] proposed a metric to estimate the quality of a camera configuration in the presence of dynamic occlusion by examining the visibility of a set of target points for a class of occluders. The occluders are vertically oriented, planar surfaces adjacent to the targets. Occluders have infinite extent so that they prevent any camera in the half-space on the opposite side of the occluder from seeing the target. Thus, the only camera locations that are un-occluded by this set of occluders lie on a vertical line that passes through the target point. For our application, these are typically not available for camera placement because the cameras would either be in the space or in the path of the projector (i.e. cast a shadow on the front protected image on the floor). Thus, with the exception of these unusable placement locations, the vertical occluders account for all possible occluders and for all possible camera locations.

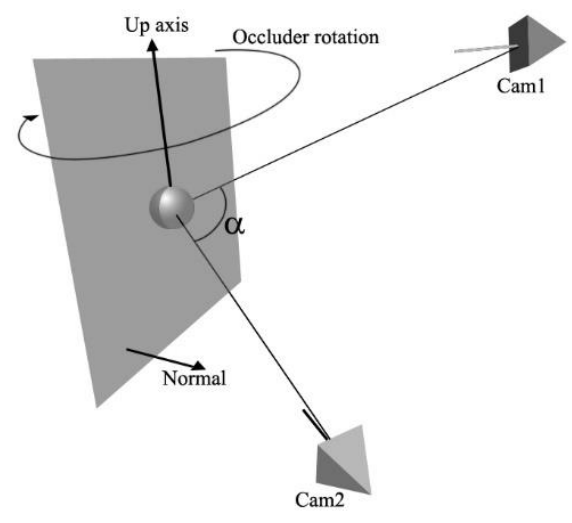

Figure 7: Perspective view of a target point and two cameras located on the front-side of an occluder.

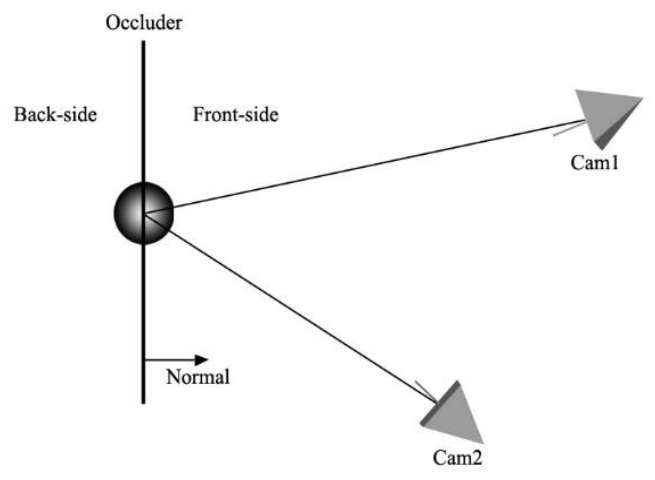

Figure 8: Top-down view of the target point and two cameras.

Such an occluder splits the volume into two half-spaces. For a given camera placement, the metric estimates the probability that at least two cameras can observe a feature point. The Chen \& Davis method draws $m$ random samples from the space of all possible occluders to estimate the expected probability of occlusion at a point. To evaluate the quality of a given camera configuration, the probability of occlusion is estimated for a sampling of all possible feature locations.

The Chen \& Davis method fails to consider two key sources of error: (1) targets that are too far from the camera to be resolved (i.e. out of range) and (2) large error propagation in the triangulation that occurs when two cameras have parallel or nearly parallel view vectors.

We propose a method that takes into account both triangulability and resolution degradation. In addition, we consider all possible occluder angles instead of a random sampling of possible occluders. Our method borrows the concept of an infinite occluder from the Chen \& Davis. The metric returns a number between 0 and 360 , representing the sum of occluder orientations for which a target is not visible in at least two triangulable views.

Calculating visibility for every single occluder rotation is not an efficient solution and it may not be feasible for large numbers of target points and cameras. Our proposed quality metric includes an efficient algorithm to calculate visibility of a target point for all occluder orientations by grouping orientations into regions of equivalent visibility.

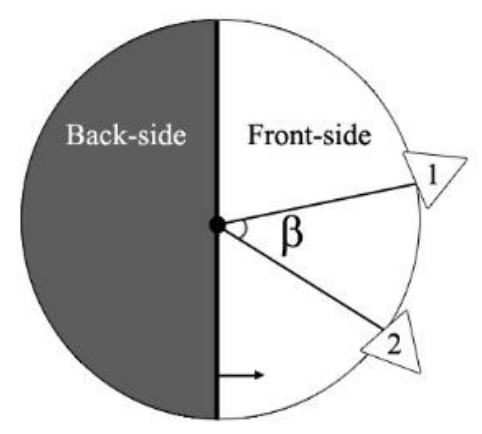

Figure 9: Simplified top-down view of the target point and two cameras. The invisible half-space is shaded and $\beta$ is the projection of the angle between view vectors of cameras onto the horizontal plane. 


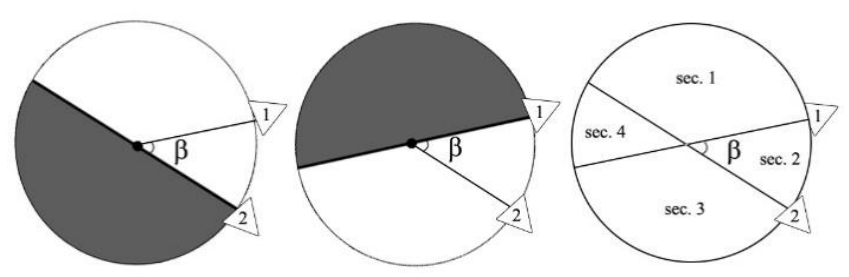

Figure 10: (Left) The occluder is very close to view vector of Cam 2, but still two triangulable cameras are covering the sample point. (Middle) Occluder is rotated clockwise to Cam 2 view vector. The left and middle figures show the range of occluder orientations over which the target point is visible by these two cameras. (Right) the extension of view vectors splits the top-down view to 4 sections.

\subsection{Quality Metric}

This section presents a quality metric based on the visibility of a target point in a least two triangulable views in the presence of dynamic occlusion. We consider all possible orientations of a vertical occluding surface and compute the sum of angles for which the target point is not visible in two triangulable views. In the next section, we use this metric in a simulated annealing algorithm to compute optimal camera placements for $n$ cameras given a set of possible target locations.

Figure 7 illustrates a 3D perspective view of a target point, the occluder, and two cameras. The target point has no volume. However, for clarity of presentation we show it as a small sphere. The occluder passes through the target point and it splits the volume into two half-spaces consisting of a front-side and a back-side. The target is occluded from view for all cameras on the back-side; the occluder does not interfere with the visibility of the target for cameras on the front-side. As the occluder rotates about the updirection, cameras change from the front side to the back side of the occluder and from the back side to the front side of the occluder. Let's assume that the target is within the effective ranges and in the fields of view of both cameras. As it is shown in the Figure 7, both cameras are located on front-side of the occluder. If the convergence angle $\alpha$ is triangulable, then this target point can be reconstructed for this occluder orientation. If there are not two such cameras on the front side, then the target cannot be reconstructed with the occluder at this orientation and the occluder angle must be added to the error metric.

Figure 8 presents a top-down view of camera arrangement in Figure 7 with the cameras placed at a constant distance from the target. This preserves the angles of view and illustrates how the orientation of the occluder influences visibility for the camera pair. The angle $\beta$ is the projection of the convergence angle between the cameras onto the horizontal plane.

Note that as the occluder rotates around up-direction, the visibility of the target point remains the same until it passes a view vector of one of the cameras. As the occluder crosses one of the view vectors, a single camera moves from the front-side to the back-side or from the back-side to the front-side. Therefore, the visibility (and triangulability) of the target is the same for all angles of the occluder between these crossings and we need only test visibility for one orientation in this range. We assume that the angle of convergence for the two cameras shown in Figure 9 is in the acceptable range for triangulation, $(40 \leq \alpha \leq 140)$, and that the target is within the effective range of both cameras. The occluder can rotate $180-\beta$ degrees clockwise from the view vector of
Cam 2 to view vector of $\operatorname{Cam} 1$ while the target point is visible for both cameras (see Figure 10). The target cannot be triangulated for other orientations of the occluder. Thus, for this pair of cameras, the occluder cannot be triangulated over a range of $180+\beta$ degrees.

Because the triangulability of a point is constant until an occluder passes the view vector of a camera, it is not necessary to enumerate all orientations of the occluder to compute the quality metric for a pair of cameras. We only need to examine one instance from each section (see Figure 10). Given $n$ cameras looking at the target point, their view vectors divide the circle of all possible occluder orientations into $2 n$ sections over which visibility is the same for all orientations within each section. We use the middle orientation of the range to test for triangulability - this orientation bisects two camera view vectors. Let $k$ be the number of cameras on the frontside of the occluder. There are $C\left(\begin{array}{l}k \\ 2\end{array}\right)$ pairs of cameras that can potentially satisfy the triangulability constraint. It is sufficient to have a single pair of cameras that satisfy the triangulability constraint in a section.

The algorithm below calculates the quality metric for a single target point. Each section (between two view vectors) is considered. For section $i$, if there is no pair of cameras satisfying the triangulability constraint, then we add the range of section $i$ to the error metric:

$C_{n}:$ Set of $n$ cameras to be placed.

$C_{k}$ : Set of $k$ cameras located on front-side of the occluder where $C_{k} \subseteq C_{n}$.

$\operatorname{Sec}_{i}$ : Angular size of section $i$. (Note $\operatorname{Sec}_{i}=\operatorname{Sec}_{i+n}$ ).

$Q$ : Sum of angles for which the current target point is not visible in two triangulable views $(0 \leq Q \leq 360)$.

$\mathrm{Cam}_{i}$ : A pair of cameras.

Flag: Set to true when the target point is visible in at least two cameras with triangulable views.

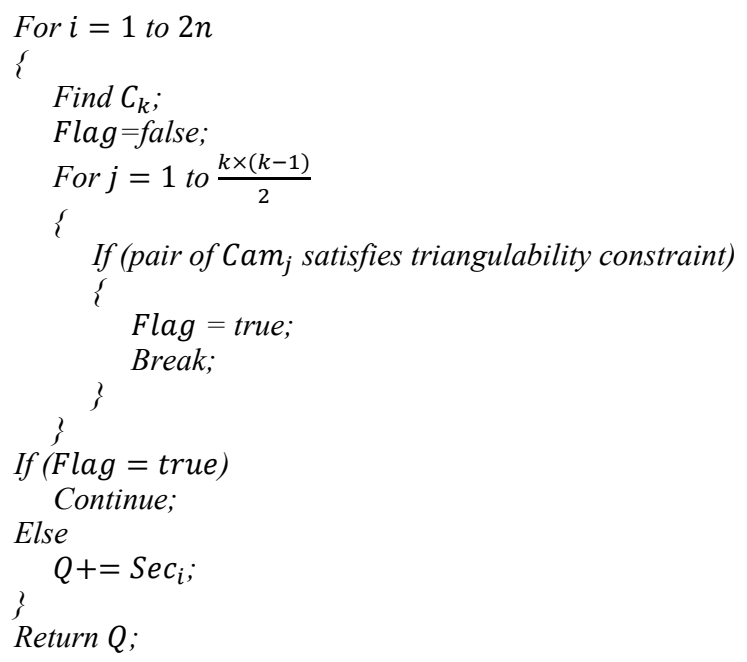

\subsection{Simulated Annealing}

Simulated Annealing (SA) is an effective method for finding a solution close to the global optimal solution [Brooks and Morgan 1995; Ingber 1993]. SA iteratively explores the solution space by varying components of the solution vector. It has been shown to produce very good, but not provably optimal results in a reasonable time for a variety of problems [Van Laarhoven and Aarts 1987]. Thus, our goal is to find a configuration that is very good, but not necessarily optimal. 
The algorithm randomly picks a camera and changes its position or/and orientation at each step. The new configuration is evaluated using the proposed quality metric. If the quality metric of the new configuration is better than the best configuration found so far, then the algorithm accepts the new configuration. On the other hand, if the new configuration is worse than the best configuration, SA accepts the new configuration with some probability, depending on the current temperature and Boltzmann selection [Mahfoud 2000]. SA accepts a configuration with a quality metric less than the best known configuration with higher probability at the beginning of the run to escape potential local minima. As the algorithm proceeds, the probability of accepting a configuration with a quality metric that is lower than that of the best known configuration is reduced until the algorithm only accepts solutions that are better than the best current configuration.

The triangulation constraint creates a challenge for SA. At least two cameras are needed to satisfy the triangulation constraint. SA changes only one camera at a time. When SA changes one camera to an uncovered area, there is no reduction in the quality metric. Because there is no observable benefit to placing a camera to cover an uncovered area, cameras convergence to one spot and the rest of volume remains uncovered.

There are two ways to address this problem. One approach is to modify the update step in SA to adjust two cameras instead of one. However, because it is highly unlikely that a random adjustment will result in placing the two cameras such that they converge on an uncovered region, this approach does little to improve the convergence of SA to a desirable solution. The other approach is to increase the penalty for uncovered target points so that SA is rewarded for adjusting a single camera to cover an uncovered region. This is the approach we've successfully implemented and tested.

For a given target point $p_{i}$, let $C_{p_{i}}$ be the set of cameras such that $p_{i}$ is in their fields of view and within their effective ranges. The new error metric, $E_{i}$, that takes into account the original quality metric and includes a penalty value $\theta$ for points that are viewable by no cameras:

$$
E_{i}= \begin{cases}360+\theta & \left|C_{p_{i}}\right|=0 \\ 360 & \left|C_{p_{i}}\right|=1 \\ Q_{i} & \left|C_{p_{i}}\right|>1\end{cases}
$$

The cost function of a given configuration $c$ is the accumulation of individual errors of all $n$ target points:

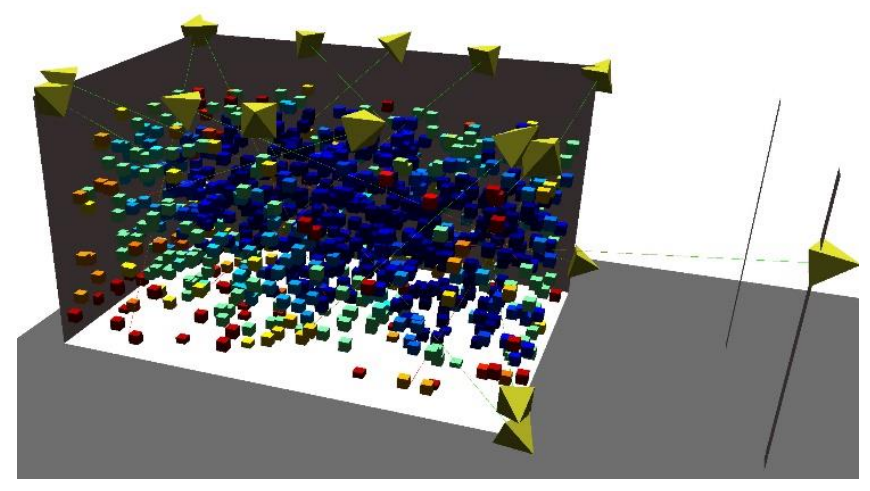

$$
f_{c}=\sum_{i=0}^{n} E_{i}
$$

To motivate SA to spread cameras across the volume, uncovered target points should have a strictly larger error than target points covered by one camera. Therefore, $\theta$ should be strictly larger than zero. Based on our experimental results, any value of $\theta$ less than 180 leads to a spread of cameras across the volume.

\section{Testing the Method}

The method was tested with both synthetically generated target point sets and target points sets captured from a person wearing a body suit covered with reflective markers walking in our CAVE virtual environment. For each point set, we computed an optimal configuration using the SA algorithm. We show the results quantitatively by reporting the average coverage (the angular range over which a target point is visible in at least two triangulable views) and visually by rendering the target point set as a heat map showing, for each point, the coverage. For the captured point sets, we empirically compare the performance of the optimal configuration computed by the SA algorithm to an ad hoc configuration and to a configuration computed using the Chen \& Davis metric.

For all but one of these tests, we restrict the possible camera locations to mountable areas around our CAVE virtual environment. However, the method allows a user to specify an arbitrary set of $3 \mathrm{D}$ constraints on camera locations. To demonstrate this flexibility, we present a solution for one of our target point sets in which the camera constraints are relaxed, simulating a 3D arena in which cameras surround the point set.

Our CAVE displays images on three vertical screens and a floor. The screens area all $244 \mathrm{~cm}$ tall. The side screens are $433 \mathrm{~cm}$ long; the front screen is $305 \mathrm{~cm}$ wide. Cameras can be mounted on the top of the screen frames and on two vertical supports at the open end of the CAVE. Mount arms give a $20 \times 20 \times 20 \mathrm{~cm}$ volume around the connection point over which the camera can be aimed in any direction. Therefore, we have a narrow volume around the top of the CAVE and both sides of the open end to place cameras. In addition, cameras can be mounted on the wall surface at the open of the CAVE.

Seventeen Optitrack Flex13 mocap cameras were used for this experiment. The Flex 13 camera has a field of view of $48^{\circ}$ and an effective range of about $365 \mathrm{~cm}$. These values were used to determine the visibility of a target point by a single camera.

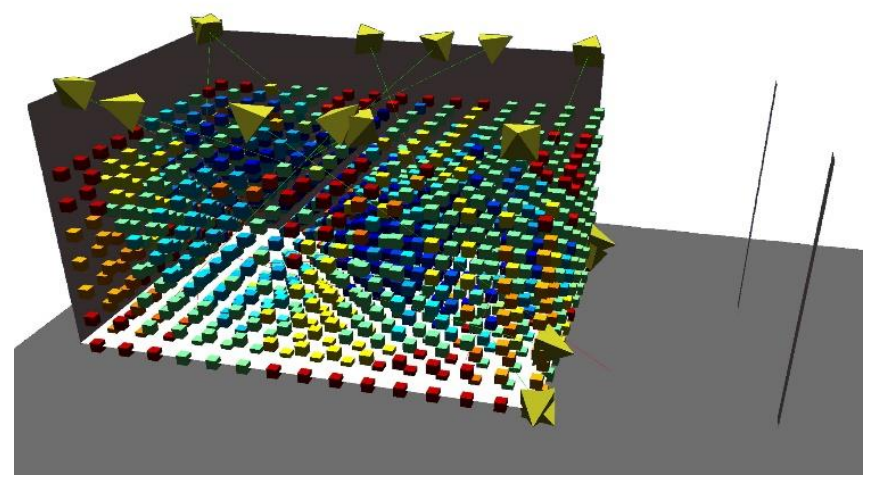

Figure 11: (Left) CAVE optimal configuration for a random sample of 1000 target points drawn from a normal distribution. (Right) CAVE optimal configuration for a uniform grid of 1155 target points. 


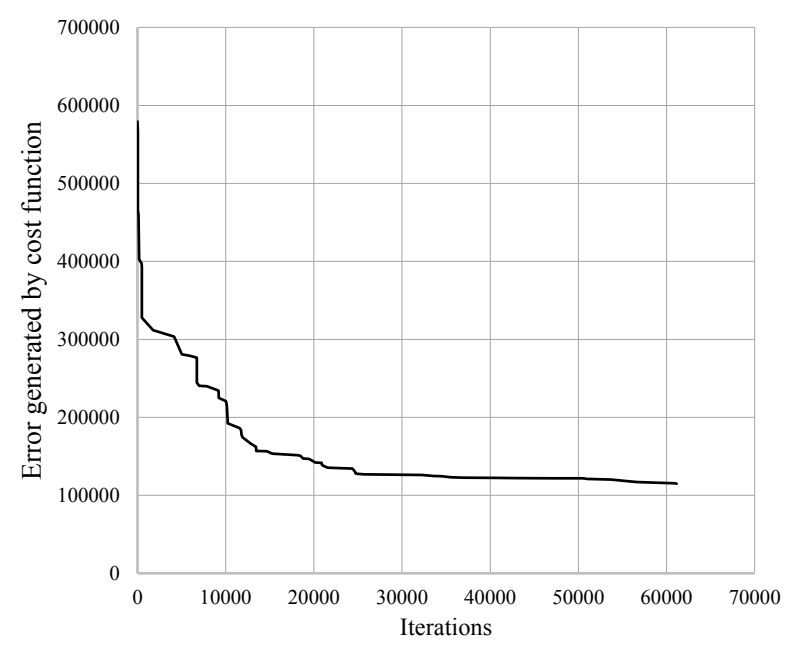

Figure 12: Average error in the optimal configuration for different numbers of cameras using a random sample of 1000 target points drawn from a normal distribution.

Triangulability was based on a target point being visible to two cameras with a convergence angle in the range $40^{\circ}-140^{\circ}$.

The tool's GUI represents the best camera placement found so far. Each cube in the scene represents a target point in the volume and its color depicts the coverage of the target point. Target points colored red are visible by at most one camera over all occluder angles; target points colored dark blue are visible by at least two triangulable views over all 360 degrees of occluder orientation. Colors between these extremes represent the magnitude of the average error metric.

\subsection{Different target distributions and CAVE}

The optimal placements for a set of 17 cameras were estimated for a $300 \times 180 \times 420 \mathrm{~cm}$ volume inside of the CAVE for two distributions of target points (see Figure 11):

a) A uniform grid of target points that filled the interior volume of the CAVE. Target points were spaced every $30 \mathrm{~cm}$ on the grid for a total of 1155 target points.

b) A random sample of target points that were uniformly distributed in the vertical and longitudinal directions and normally distributed about the midline of CAVE in the lateral (side to side) direction. This placed more points near the center of the CAVE where participants are more likely to be in our experiments. Target points were distributed throughout the volume of the CAVE.

Table 1: Average of invisibility for uniform grid and a sample of target points drawn from a normal distribution.

\begin{tabular}{|l|c|c|l|}
\hline Distribution & $\begin{array}{c}\text { \# of target } \\
\text { points }\end{array}$ & $\begin{array}{c}\text { Avg. of invisibility } \\
\text { (degree) }\end{array}$ & S.D. \\
\hline Uniform & 1155 & 183.09 & 10.84 \\
\hline Normal & 1000 & 101.82 & 2.95 \\
\hline
\end{tabular}

Table 1 presents the visibility metric (i.e. range of orientations for which targets points are not visible in two triangulable views) in the optimal camera configuration computed by SA for the two target point sets: a uniform grid and a sample drawn from a normal distribution about the midline of the CAVE. The corresponding heat map renderings of target point visibility are displayed in Figures 11.

The best coverage is achieved with the normal distribution. As is apparent in the heat map rendering, the most difficult areas to cover lie near the screens. Because of the constraints on camera locations, it is difficult to place cameras to see target points from all sides when the targets are close to a screen. A greater proportion of target points lie near with midline of CAVE in the normal distribution and, hence, the average performance is superior to that achieved with the uniform distribution.

Figure 12 shows the rate of convergence over 62,000 iterations of the SA algorithm. The example shown was based on a normal distribution described above. However, a similar pattern of convergence was observed for other target point sets. The error dropped very quickly at the beginning of SA process and stabilized after about 25,000 iterations. The running time for this example took about 5 hours to accomplish over 62,000 iterations of the SA algorithm; however, the convergence was stable after about 2 hours.

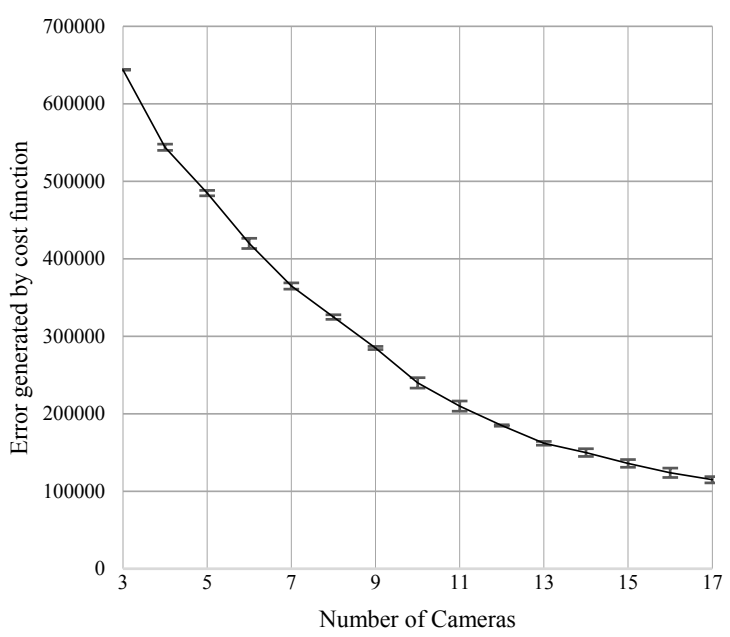

Figure 13: Average error in the optimal configuration for different numbers of cameras using a random sample of 1000 target points drawn from a normal distribution.

To examine how the number of cameras influenced the size of the error in the final result, we ran the SA algorithm for camera sets of size 3 to 17. As would be expected, the larger the number of cameras, the better the result. Figure 13 gives the minimum error achieved for different numbers of cameras. The results are based on the sample set of normally distributed target points used in Table 1. Each configuration was repeated 10 times. The error bar represents the standard deviation (SD). A similar pattern of results was obtained for other data sets. The error was reduced as the number of cameras was increased but the improvement decreased as additional cameras were added.

\subsection{Empirical results}

An important motivation for our work is to place cameras to optimally recover full-body motions in the CAVE. To examine the suitability of the algorithm for our application, we generated a 


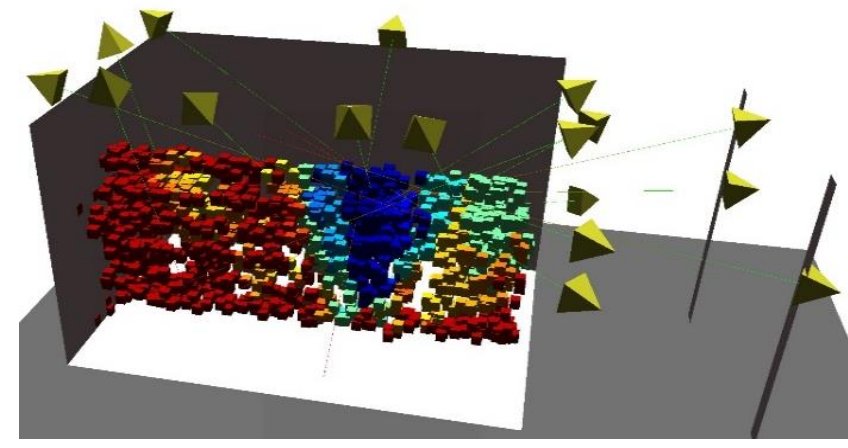

(A. Ad hoc configuration)

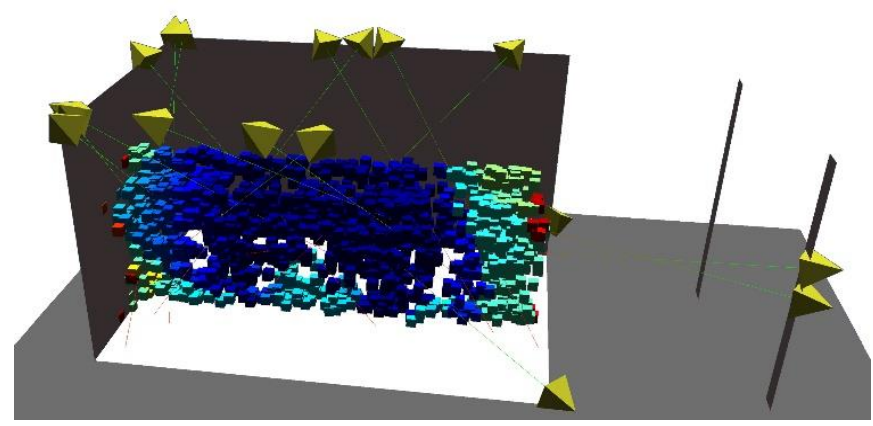

(B. CAVE Chen \& Davis configuration)

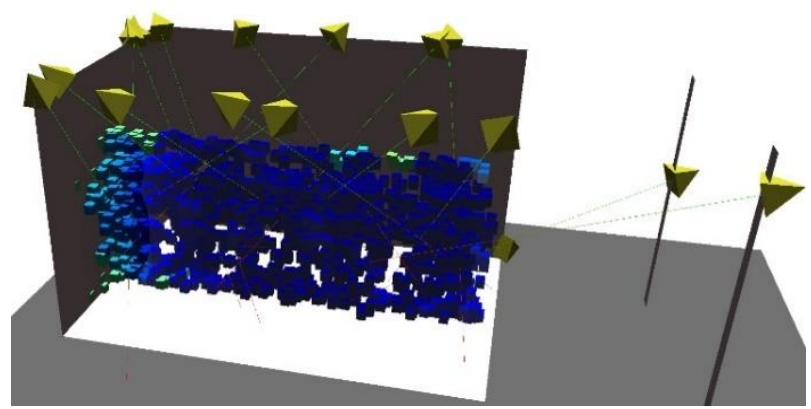

(C. CAVE proposed configuration)

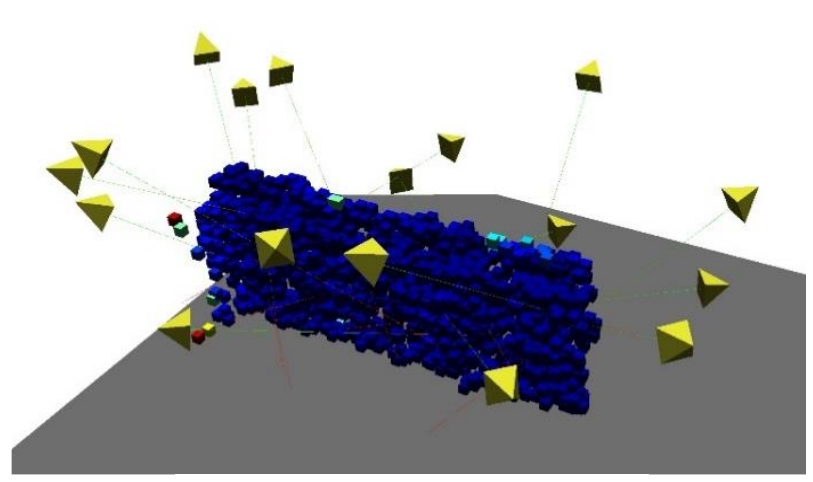

(D. Arena optimal configuration)

Figure 14: Heat maps of visibility of target points drawn from motion capture of walking in the CAVE for different camera configurations. Red means no or poor coverage whereas blue indicates high coverage with low invisibility. target point distribution from actual walking motions. We then computed the optimal camera placements using the SA algorithm with the proposed metric and again with the Chen \& Davis metric. We report the value of the visibility metric for the configurations returned and for an ad hoc configuration. Finally, we placed the cameras in the three different configurations (ad hoc, Chen \& Davis, and proposed) and examined the performance for live motion capture.

Target points for real walking were captured from a participant wearing a body suit with 37 markers attached. The cameras were placed in an ad hoc arrangement for this initial capture. The participant walked from the back to the front of the CAVE along the midline for $2 \mathrm{~min}$. The Motive software was used to capture the locations of the target points at $120 \mathrm{fps}$. Two minutes of walking generated 14,400 frames. Every frame could contain at most 37 markers, but due to the sub-optimal camera placement, most of frames had fewer than 37 matches. In order to have a manageable number of target points, the raw distribution was sub-sampled by selecting every 240th frame. The selected frames gave a fair representation of the movement. To further distribute the captured data, we shifted the $3 \mathrm{D}$ position of all points in each frame with a randomly selected frame. From this process we extracted 1659 target points that were then used to determine the optimal camera placements.

For many applications (e.g. character animation), full-body motions are captured in an open environment that is not confined by the screens of the CAVE. This allows cameras to surround the capture volume and offers greater visibility as compared to a CAVE. To examine how the camera placement constraints for a CAVE influence visibility, the same set of target points were employed to find the optimal camera placement for an arena. For the arena, cameras were constrained to lie in a volume enclosing the CAVE created by expanding the dimensions of the sides of the CAVE outward by one meter (essentially forming an envelope surrounding the CAVE). We expect the optimal configuration of the arena to outperform the optimal camera configuration for a CAVE.

Table 2: Average of invisibility for a sample of $3 D$ points captured from walking in the CAVE.

\begin{tabular}{|l|c|c|l|}
\hline Configuration & $\begin{array}{c}\text { \# of } \\
\text { target } \\
\text { points }\end{array}$ & $\begin{array}{c}\text { Avg. of } \\
\text { invisibility } \\
\text { (degree) }\end{array}$ & S.D. \\
\hline CAVE ad hoc & 1659 & 254.93 & N/A \\
\hline CAVE Chen \& Davis & 1659 & 69.15 & 9.31 \\
\hline CAVE Proposed & 1659 & 27.91 & 3.73 \\
\hline Arena Proposed & 1659 & 7.44 & 1.69 \\
\hline
\end{tabular}

Table 2 presents the visibility metric for the ad hoc configuration, the optimal configuration computed using the Chen \& Davis metric, and the optimal configuration computed with the proposed metric (all for a CAVE setup). For comparison, the visibility metric for the proposed method in an arena setup is also shown. The optimization results are all based on 10 runs of the SA algorithm. The corresponding heat map renderings of target point visibility are displayed in Figure 14. As expected, the best performance was achieved with the arena configuration. The proposed method outperformed the ad hoc placement and the configuration computed with the Chen \& Davis metric in the CAVE setup. For all the methods, the most problematic areas in the CAVE are positions near the front of the screen. 
Table 3: Full-body motion capture results for walking along the midline of the CAVE.

\begin{tabular}{|l|c|c|c|}
\hline & $\begin{array}{c}\text { Ad } \\
\text { hoc }\end{array}$ & $\begin{array}{c}\text { Chen } \\
\text { \& } \\
\text { Davis }\end{array}$ & Proposed \\
\hline $\begin{array}{l}\text { Average of Unmatched } \\
\text { markers }\end{array}$ & 1.52 & 0.42 & 0.29 \\
\hline $\begin{array}{l}\text { Number of frames where } \\
\text { full body detected (total } \\
\text { frames=14,400) }\end{array}$ & 4322 & 10122 & 12346 \\
\hline
\end{tabular}

Our final test of the effectiveness of the computed optimal configuration was to examine how well it performed with real-time capture of full-body motions. For this, we physically moved our cameras to the locations identified as optimal by the SA algorithm. We then captured walking motions with the optimally configured cameras and compared performance with the ad hoc configuration and the cameras positioned in the configuration computed with the Chen \& Davis metric.

To facilitate physical camera placement, the optimization tool produces a map of camera placements. In addition, the tool computes the projection of the central axis of the camera and where it intersects a screen of the CAVE. Based on this projection, the tool produces an image showing the screen intersections that can be displayed on a wall of the CAVE. These markings serve as reference points for aiming the cameras once they are correctly positioned. When a camera is correctly positioned, it can be oriented by centering the corresponding intersection point on the camera image.

To test the effectiveness of the camera configuration in capturing full-body motion, we used the Motive software to capture 2 minutes of walking along the midline of the CAVE. Motions were separately captured with the cameras in the original ad hoc placement, in the configuration computed by the Chen \& Davis method, and in the computed optimal placement. A participant wore a body suit with 37 retro-reflective markers on it. A total of 14,400 frames of data were collected with each camera configuration. To evaluate the capture, we look at the number of frames in which all 37 markers are successfully matched to the skeletal model and the average number of unmatched markers on each frame. As shown in Table 3, the optimal configuration matched all 37 markers on 12,347 or $86 \%$ of the frames; the Chen \& Davis method achieved $70 \%$ accuracy by successfully detecting 10122 frames; and the ad hoc configuration matched all 37 markers

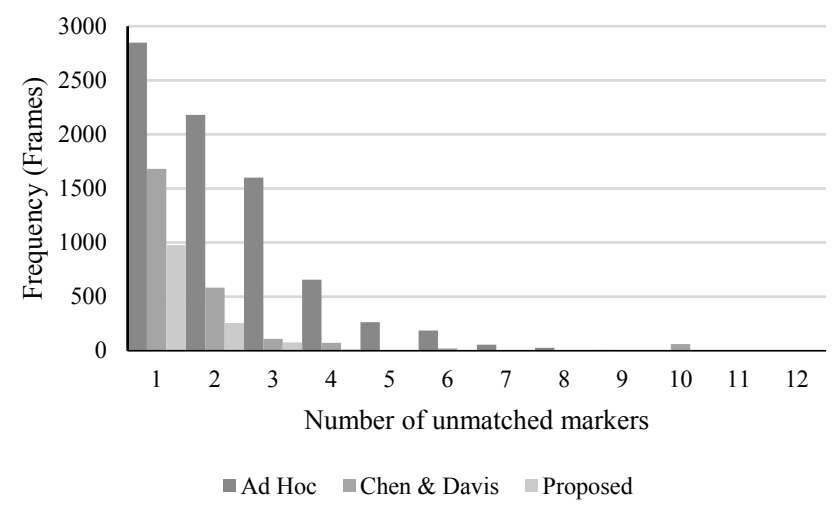

Figure 15: Histogram of frequency of unmatched markers for 3 different methods. on 4322 or $30 \%$ of the frames. The average number of unmatched markers reflects this disparity in performance $(0.29$ unmatched markers per frame for the optimal configuration, 0.42 unmatched markers per frame for the Chen \& Davis configuration, and 1.5 unmatched markers per frame for the ad hoc configuration).

Fig. 15 shows the frequency of occurrence of unmatched markers for the ad hoc configuration, the optimal configuration computed with the Chen \& Davis metric, and the optimal configuration computed using the proposed metric. The configuration computed by the proposed method had the fewest number of unmatched markers. The configuration computed with the Chen \& Davis metric had the next fewest number of unmatched markers. However, in some frames as many as 12 markers were missed. The ad hoc configuration had the largest number of unmatched markers.

\section{Conclusion}

This paper proposes a method to optimally position a set of cameras to capture motions in the presence of dynamic occlusion. The method balances error propagation properties of triangulation computations and visibility for a class of occluders (vertical planes adjacent to a target point). The goal is to find a "sweet spot" where targets are likely to be visible in two triangulable cameras (i.e. cameras with view vectors that can be triangulated with minimal error). The approach allows designers to select a collection of target locations based on their understanding of the application and where targets are likely to be. Designers can also select the number of cameras and specify constraints on where the cameras can be located.

The results demonstrate that the configuration computed by the optimization algorithm can lead to substantial performance gains over ad hoc camera placement and a configuration based on the Chen \& Davis method both in terms of the visibility metric and in the robustness of full-body motion capture using a commercial software capture system.

In future work, we plan to develop a parallel version in order to accelerate the process of error estimation. The method is wellsuited for parallelization because each target point can be estimated independently. Another interesting direction for future work is to use this algorithm as a basis to estimate the minimum number of cameras for a given accuracy threshold and workspace.

\section{Acknowledgments}

This research was supported by National Science Foundation awards BCS-1251694 and CNS-1305131, and by the US Department of Transportation, Research and Innovative Technology Administration, Prime DFDA No. 20.701, Award No. DTRT13-G-UTC53.

\section{References}

BROOKS, S.P. AND Morgan, B.J. 1995. Optimization using simulated annealing. The Statistician, 241-257.

Brown, M.Z., BurschKA, D. AND Hager, G.D. 2003. Advances in computational stereo. IEEE Transactions on Pattern Analysis and Machine Intelligence 25, 8, 993-1008.

Chen, X. AND DAvis, J. 2008. An occlusion metric for selecting robust camera configurations. Machine Vision and Applications 
$19,4,217-222$.

Cruz-Neira, C.C. Sandin, D.J., Defanti, T.A., Kenyon, R.V. AND HART, J.C. 1992. The CAVE: audio visual experience automatic virtual environment. Communications of the ACM $35,64-72$.

Cowan, C.K. AND Kovesi, P.D. 1988. Automatic sensor placement

from vision task requirements. IEEE Transactions on Pattern Analysis and Machine Intelligence 10, 3, 407-416.

Fleishman, S., COHEN-OR, D. AND LisCHINSKI, D. 1999. Automatic camera placement for image-based modeling. In Proceedings Seventh Pacific Conference on Computer Graphics and Applications. IEEE, 12-20.

HARTLEy, R. AND ZisSERMAN, A. 2003. Multiple view geometry in computer vision. Cambridge University Press.

Hartley, R.I. AND Sturm, P. 1997. Triangulation. Computer vision and image understanding 68, 146-157.

INGBER, L. 1993. Simulated annealing: Practice versus theory. Mathematical and Computer Modelling 18, 11, 29-57.

Katz, I., Aghajan, H. AND Haymaker, J. 2010. A Process for Sensor Configuration in Multi-Camera Networks. In Proc. 4th ACM/IEEE Int. Conf. Distributed Smart Cameras.

LiU, B., Yu, M., MAier, D. AND MANNER, R. 2005. An efficient and accurate method for 3D-point reconstruction from multiple views. International Journal of Computer Vision 65, 2, 175-188.

Lourakis, M. AND Argyros, A. 2004. The design and implementation of a generic sparse bundle adjustment software package based on the Levenberg-Marquardt algorithm. Technical Report 340, Institute of Computer Science-FORTH, Heraklion, Crete, Greece.

MAHFoud, S.W. 2000. Boltzmann selection. In T. Bäck , D.B. Fogel,

and Z. Michalewicz, eds., Evolutionary Computation 1: Basic Algorithms and Operators. Institute of Physics Publishing, Bristol, 195-200.

MASON, S. 1997. Heuristic reasoning strategy for automated sensor placement. Photogrammetric Engineering and Remote Sensing $63,9,1093-1101$.

NuRRE, J. AND HALL, E. 1986. Error analysis for a two-camera stereo vision system. Applications of Artificial Intelligence III. SPIE-The International Society for Optical Engineering 635, 216-229.

O'ROURKE, J. 1987. Art gallery theorems and algorithms (Vol. 57). Oxford: Oxford University Press.

Olague, G. AND Mohr, R. 2002. Optimal camera placement for accurate reconstruction. Pattern Recognition 35, 4, 927-944.

Rahimian, P. AND KeARnEY, K.J. 2015. Camera Placement Tool www.myweb.uiowa.edu/rahimian/CameraPlacement.zip.
SANDERS-REED, J.N. 2001. Error propagation in two-sensor three-dimensional position estimation. Optical Engineering 40, 4 , 627-636.

SANDERS-REED, J.N. 2002. Impact of tracking system knowledge on

multisensor 3D triangulation. In AeroSense 2002. International Society for Optics and Photonics, 33-41.

SAnders-ReED, J.N. 2002. Triangulation Position Error Analysis for

Closely Spaced Imagers (No. 2002-01-0685). SAE Technical Paper.

SHERMER, T.C. 1992. Recent results in art galleries [geometry]. Proceedings of the IEEE 80, 1384-1399.

VAN LAARHOVEN, P.J. AND AARTS, E.H. 1987. Simulated annealing: theory and applications. Springer Science \& Business Media.

WU, J.J., Sharma, R. AND HuAnG, T.S. 1998. Analysis of uncertainty bounds due to quantization for three-dimensional position estimation using multiple cameras. Optical Engineering $37,1,280-292$. 